\title{
Global Coupling and Decoupling of the APS Storage Ring
}

\author{
Yong-chul Chae, Jianyang Liu, Lee C. Teng \\ APS, Argonne National Laboratory
}

\section{Introduction}

This paper describes a study of controlling the coupling between the horizontal and the vertical betatron oscillations in the APS storage ring. First, we investigate the strengthening of coupling using two families of skew quadrupoles. Using smooth approximation, we obtained the formulae to estimate the coupling ratio defined as the ratio of the vertical and horizontal emittances or, for a single particle, the ratio of the maximum values of the Courant- Snyder invariants. Since we knew that the coupling is mostly enhanced by the $21^{s t}$ harmonic content of skew quadrupole distribution, we carried out the harmonic analysis in order to find the optimum arrangement of the skew quadrupoles. The numerical results from tracking a single particle are presented for the various configurations of skew quadrupoles. Second, we describe the global decoupling procedure to minimize the unwanted coupling effects. These are mainly due to the random roll errors of normal quadrupoles. It is shown that even with the rather large rms roll error of $2 \mathrm{mrad}$ we can reduce the coupling from 70 percent to 10 percent with a skew quadrupole strength which is one order of magnitude lower than the typical normal quadrupole strength.

\section{Global Coupling}

\subsection{Treatment of Weak Coupling Using Smooth Approximation}

The Hamiltonian including the skew quadrupoles is

$$
H=\frac{1}{2}\left(p_{x}^{2}+p_{y}^{2}\right)+\frac{1}{2} K(s)\left(x^{2}-y^{2}\right)+M(s) x y,
$$

where $K(s)=\frac{1}{(B \rho)} \frac{\partial B_{y}}{\partial x}$ and $M(s)=\frac{1}{(B \rho)} \frac{\partial B_{y}}{\partial y}$. The equation of motion with $\theta=\frac{s}{R}$ as the independent variable is

$$
\begin{gathered}
x^{\prime \prime}+k(\theta) x=-m(\theta) y \\
y^{\prime \prime}-k(\theta) y=-m(\theta) x,
\end{gathered}
$$

where $k(\theta)=R^{2} K(s), m(\theta)=R^{2} M(s)$ and prime denotes $\frac{d}{d \theta}$. To analyze the above linear coupled equation, we may use the "smooth" approximation ([1], [2]). Let

$$
\begin{aligned}
& x(\theta)=X(\theta)+u(X, \theta) \\
& y(\theta)=Y(\theta)+v(Y, \theta),
\end{aligned}
$$




\section{DISCLAIMER}

This report was prepared as an account of work sponsored by an agency of the United States Government. Neither the United States Government nor any agency thereof, nor any of their employees, make any warranty, express or implied, or assumes any legal liability or responsibility for the accuracy, completeness, or usefulness of any information, apparatus, product, or process disclosed, or represents that its use would not infringe privately owned rights. Reference herein to any specific commercial product, process, or service by trade name, trademark, manufacturer, or otherwise does not necessarily constitute or imply its endorsement, recommendation, or favoring by the United States Government or any agency thereof. The views and opinions of authors expressed herein do not necessarily state or reflect those of the United States Government or any agency thereof. 


\section{DISCLAIMER}

Portions of this document may be illegible in electronic image products. Images are produced from the best available original document. 
where the "smooth" variable $X(\theta)$ is a slowly varying function of $\theta$, and $u(X, \theta)$ is rippling with the period of $2 \pi$ and its average is zero. Then the equation of motion for the smooth variables can be written

$$
\begin{gathered}
X^{\prime \prime}+\nu_{x}^{2} X=-m(\theta) Y \\
Y^{\prime \prime}+\nu_{y}^{2} Y=-m(\theta) X
\end{gathered}
$$

where $\nu_{x, y}$ are the tunes but in the context of smooth approximation, it is the mean restoring force defined as

$$
<k>+<\left(\int(k-<k>) d \theta\right)^{2}>\cong \nu^{2}
$$

for uncoupled linear betatron oscillation.

Assuming weak coupling, the solution of Eq. (4) may be obtained by substituting the homogeneous solution of $X$ (or $Y$ ) into the right-hand side of Eq. (4). To simplify the calculation, we assume the initial conditions at $\theta=0$ to be

$$
X=1, X^{\prime}=0, Y=0 \text { and } Y^{\prime}=0 .
$$

Then, substituting $X=\cos _{x} \theta$ into the equation for $\dot{Y}$ we get

$$
Y^{\prime \prime}+\nu_{y}^{2} Y=-m(\theta) \cos \nu_{x} \theta
$$

Expanding $m(\theta)$ in the Fourier series

$$
-m(\theta)=\sum_{k=-\infty}^{k=+\infty}\left(a_{k} \cos k \theta+b_{k} \sin k \theta\right)
$$

we obtain the solution for $Y$

$$
Y=\sum_{k=-\infty}^{k=+\infty} \frac{\frac{1}{2}\left(a_{k} \cos \left(\nu_{x}-k\right) \theta-b_{k} \sin \left(\nu_{x}-k\right) \theta\right)}{\nu_{y}^{2}-\left(\nu_{x}-k\right)^{2}}
$$

If the tunes are near the coupling resonance, $\nu_{x}-\nu_{y} \cong k$,

$$
Y_{A m p} \cong \frac{\frac{1}{2} \sqrt{a_{k}^{2}+b_{k}^{2}}}{\nu_{y}^{2}-\left(\nu_{x}-k\right)^{2}}
$$

and the coupling ratio becomes

$$
\kappa=\frac{\epsilon_{y}}{\epsilon_{x}}=\frac{Y_{A m p}^{2}}{X_{A m p}^{2}} \propto\left(a_{k}^{2}+b_{k}^{2}\right) .
$$

For the APS storage ring, the tunes are $\nu_{x}=35.22$ and $\nu_{y}=14.30$. Hence, the $21^{\text {st }}$ harmonic of skew quadrupole distribution around the ring causes the coupling most efficiently. In the next sections, the arrangement of skew quadrupoles to excite the $21^{\text {st }}$ harmonic is discussed and some numerical and analytical results are presented. 


\subsection{Arrangement of Skew Quadrupoles}

Consider $\mathrm{N}$ skew quadrupoles with the same strength evenly distributed around the ring with period $\frac{2 \pi}{N}$ as shown in Fig. 1-a. Then

$$
f_{1}(\theta)=\sum_{n=-\infty}^{n=+\infty} \delta\left(\theta-\frac{2 \pi}{N} n\right)=\frac{N}{2 \pi}+\frac{N}{\pi} \sum_{n=-\infty}^{n=+\infty} \cos (n N \theta) .
$$

Fourier harmonic numbers are $k=n N$ where $n$ is an integer. In order to control the harmonic number $k$ such that $k=n N+m M$, we may apply on top of $f_{1}(\theta)$ the periodic staircase functions whose period equals $\frac{2 \pi}{M}$. Such functions are shown in Fig. 1-b and

$$
f_{2}(\theta)=\sum b_{m} \sin (m \dot{M} \theta)
$$

where $b_{m}=\frac{4}{m \pi}$ and $m=$ odd integer. Thus,

$$
\begin{aligned}
f(\theta) & =f_{1}(\theta) f_{2}(\theta) \\
& =\frac{2 N}{\pi^{2}} \sum_{m} \frac{\sin (m M \theta)}{m}+\frac{2 N}{\pi^{2}} \sum_{n} \sum_{m} \frac{\sin (n N+m M) \theta-\sin (n N-m M) \theta}{m} .
\end{aligned}
$$

Hence we show that we can generate an arbitrary harmonic by changing the period of the staircase functions.

In the APS storage ring, the spaces available for the skew quadrupoles are between Q3 and S2 in the upstream half of a sector (half sector A), which we will call the A:QS family, and between Q4 and S3 in the downstream half of a sector (half sector B), which we will call the B:QS family. This arrangement is shown in Fig. 2 which illustrates the nomenclature rules [3]. The number of skew quadrupoles considered is ten for each family. We may install the focusing A:QS in every fourth cell, say cell numbers $1,5,9,13$ and 17 , and the defocusing A:QS in cells $21,25,29,33$ and 37 . This family alone can adequately generate the desired $21^{\text {st }}$ harmonic. Using Eq. (8), with $N=10$ and $M=1$ for the A:QS family, we find the coefficient of the $21^{\text {st }}$ harmonic to be $b_{21} \equiv c=\frac{20}{\pi^{2}}$ which is greater than unity. When the additional B:QS family is added to the ring, the $21^{\text {st }}$ harmonic coefficient has the interference term because $A: Q S$ and $B: Q S$ are not in phase. For the arrangement shown in Fig. 1-c which we will call the "normal" arrangement, we may write

$$
-m(\theta)=c(a \cos 21 \theta+b \sin 21 \theta)
$$

where $a=-\sin 21 \Delta \theta_{o}, b=1+\cos 21 \Delta \theta_{o}$, and $\Delta \theta_{o}$ is the shift of the origin of the B:QS family with respect to the origin of the A:QS family which is the middle of the A:QS skew quadrupole in cell number 1 . In the APS storage ring $\Delta \theta_{0}$ is $\frac{\pi}{8}$. We note that, if $A: Q S$ and $\mathrm{B}: \mathrm{QS}$ are exactly in phase, $a=0$ and $b=2$.

In the next section, we present numerical results of the coupling coefficient obtained by tracking a single particle. We first use the "normal" arrangement as the basis and then we attempt to find the optimum arrangement for obtaining full coupling, $\kappa=1$. 


\subsection{Numerical Results}

For the single particle motion the Courant-Snyder invariant is

$$
\epsilon_{x}=\frac{x^{2}+\left(\alpha_{x} x+\beta_{x} x^{\prime}\right)^{2}}{\beta_{x}}
$$

The coupling ratio in this report is defined as

$$
\kappa=\frac{\left(\epsilon_{y}\right)_{\max }}{\left(\epsilon_{x}\right)_{\max }}
$$

This definition is consistent with the ratio of emittances of a group of particles (a beam), because the emittance is the phase space area enclosed by the envelope of the beam. However, since the linear optical parameters, $\beta_{x, y}$ and $\alpha_{x, y}$, are ill-defined in the coupled lattice, our definition of the emittance is not the true projection of the four-dimensional phase space volume onto the $\mathrm{x}$ or $\mathrm{y}$ plane as defined in Ref. [4]. But for our application it is an adequate approximation to the real projected emittance.

In order to estimate the coupling ratio with the intentional insertion of skew quadrupoles in the otherwise uncoupled APS storage ring lattice, we used the programs MAD [5] and RACETRACK [6]. The phase space structures obtained from the two programs are in good agreement. The results shown in this report are those from the program MAD. The coupling ratios for the various configurations are summarized in Table 1 . The first column shows which family of skew quadrupole is excited to couple the beam. Each family consists of ten skew quadrupoles. For full coupling, $\kappa=1$, we found that A:QS is 20 percent more efficient than B:QS. This is because $\beta_{x}=7.52 m$ and $\beta_{y}=13.63 m$ at A:QS, $\beta_{x}=3.54 m$ and $\beta_{y}=20.50 m$ at $\mathrm{B}: \mathrm{QS}$, and the coupling is proportional to $\sqrt{\beta_{x} \beta_{y}}$. Using both families, we could obtain full coupling with the integrated skew quadrupole strength of $B^{\prime} l=0.25 \mathrm{~T}$ which is larger than the $0.2 \mathrm{~T}$ of the design normal operating strength.

In order to achieve full coupling at the skew quadrupole strength $0.2 \mathrm{~T}$, we optimized the skew quadrupole arrangement. One optimization procedure is to rotate the B:QS family by $n \frac{2 \pi}{10}$ in clockwise direction while A:QS is fixed at the original place. With $n=1, \mathrm{~B}: \mathrm{QS}$ in cell 3 goes to cell 7 and B:QS in cell 7 to cell 11 and so on. This operation is shown in Fig. 1-d. By using this shifting operation, we control the $a$ and $b$ coefficients in Eq. (9) which can be written

$$
\begin{array}{ll}
a=a(A: Q S)+a(B: Q S), & b=b(A: Q S)+b(B: Q S) \\
a(A: Q S)=0, & b=b(A: Q S)=1 \\
a(B: Q S)=-\sin 21 \Delta \theta_{n}, & b(B: Q S)=\cos 21 \Delta \theta_{n},
\end{array}
$$

where $\Delta \theta_{n}=\Delta \theta_{0}+\frac{2 \pi}{10} n$ and $\Delta \theta_{0}=\frac{\pi}{8}$. The coefficients $a(B: Q S)$ and $b(B: Q S)$ for different $n$ values are plotted in the polar coordinate system as in Fig. 3 (top). We notice that two skew families are almost in phase when $n=7$ and the amplitude of the $21^{\text {st }}$ harmonic resulting from Fig. 3 (bottom), is

$$
\left|c_{21}\right|=\sqrt{a_{21}^{2}+b_{21}^{2}} \cong 2
$$




\begin{tabular}{|c|c|c|c|c|}
\hline Skew quadrupoles & $\overline{B^{\prime} l(T)}$ & $\epsilon_{x \max } / \epsilon_{x 0}$ & $\epsilon_{y \max } / \epsilon_{x 0}$ & $\kappa=\epsilon_{y \max } / \epsilon_{x \max }$ \\
\hline \multirow{7}{*}{$Q_{s 3}+Q_{s 4}$} & 0.30 & 2.3936 & 2.6330 & 1.1000 \\
\hline & 0.25 & 1.8577 & 1.8069 & 0.9741 \\
\hline & 0.20 & 1.4991 & 1.1874 & 0.7921 \\
\hline & 0.15 & 1.2604 & 0.7031 & 0.5579 \\
\hline & 0.10 & 1.1027 & 0.3195 & 0.2897 \\
\hline & 0.05 & 1.0260 & 0.0860 & 0.0838 \\
\hline & 0.00 & 1.0000 & 0.0000 & 0.0000 \\
\hline \multirow{7}{*}{$Q_{s 3}$} & 0.30 & 1.5513 & 1.5555 & 1.0028 \\
\hline & 0.25 & 1.3667 & 1.3057 & 0.9553 \\
\hline & 0.20 & 1.2237 & 1.0343 & 0.8452 \\
\hline & 0.15 & 1.1280 & 0.7280 & 0.6454 \\
\hline & 0.10 & 1.0555 & 0.3978 & 0.3769 \\
\hline & 0.05 & 1.0133 & 0.1168 & 0.1153 \\
\hline & 0.00 & 1.0000 & 0.0000 & 0.0000 \\
\hline \multirow{7}{*}{$Q_{s 4}$} & 0.30 & 1.2501 & 1.0835 & 0.8667 \\
\hline & 0.25 & 1.1715 & 0.8571 & 0.7317 \\
\hline & 0.20 & 1.1099 & 0.6319 & 0.5693 \\
\hline & 0.15 & 1.0636 & 0.4148 & 0.3900 \\
\hline & 0.10 & 1.0284 & 0.2116 & 0.2101 \\
\hline & 0.05 & 1.0070 & 0.0585 & 0.0581 \\
\hline & 0.00 & 1.0000 & 0.0000 & 0.0000 \\
\hline
\end{tabular}

Table 1: Effects of skew quadrupole arrangment on the linear coupling ratio

which is the desired result.

The tune separation and the coupling ratio for various arrangements of the B:QS family of the skew quadrupoles with the integrated strength $B^{\prime} l=0.2 T$ are listed in Table 2. The tune separation data, an indication of coupling, clearly shows that the $n=7$ arrangement is the most efficient way of coupling the lattice. However, the coupling ratio doesn't show a clear advantage of the $n=7$ over the $n=8$ arrangement. This is because once the beam is close to full coupling, the coupling ratio is saturated in the sense that it does not gain much advantage from the optimized arrangement to a less optimized one. This saturationbehaviour is shown in Fig. 4 (top). Also, our definition of emittance is not rigorous. Finally, the coupling ratios around the ring are plotted in Fig. 4 (bottom) for a useful comparison.

\section{Global Decoupling}

\subsection{Treatment of Weak Coupling Using Matrix Formalism}

The Courant-Snyder parameterization for one-dimensional linear motion in a periodic system is generalized to two-dimensional coupled linear motion by Edwards and Teng [7]. This work is further developed and put into a more convenient form for compensating coupling by Peggs [8]. A detailed description of the above matrix formalism can be found in Tal- 


\begin{tabular}{|c|c|c|}
\hline \hline Arrangement No. & $\left|\nu_{x}-\nu_{y}\right|$ & $\kappa=\epsilon_{\text {ymax }} / \epsilon_{\text {xmax }}$ \\
\hline 0 (normal) & 0.104 & 0.797 \\
\hline 1 & 0.080 & 0.403 \\
\hline 2 & 0.087 & 0.542 \\
\hline 3 & 0.115 & 0.798 \\
\hline 4 & 0.145 & 0.891 \\
\hline 5 & 0.171 & 0.888 \\
\hline 6 & 0.185 & 0.936 \\
\hline 7 & 0.186 & 0.952 \\
\hline 8 & 0.172 & 0.963 \\
\hline 9 & 0.145 & 0.936 \\
\hline \hline
\end{tabular}

Table 2: Optimization of skew quadrupole arrangment

man's paper [9]. Applications of this theory in a real storage ring known to us are found in the work of Billing at CESR [10]. Measurement and correction of the local coupling in the storage ring at CESR are described by Bagley and Rubin [11,12]. In addition, the global decoupling at the design stage of a ring which is relevant to this work is described by Schachinger at SSC [13].

Following Peggs, we may write the normalized transfer matrix for the ring as

$$
T=\left(\begin{array}{cc}
M & m \\
n & N
\end{array}\right) .
$$

This normalized transfer matrix is the similarity transformation of the Edwards and Teng matrix, $T^{\prime}$. The relation between the two is

$$
T=G T^{\prime} G^{-1}
$$

where $\mathrm{G}$ is the $4 \times 4$ Floquet transformation matrix defined as

$$
\begin{aligned}
G & =\left(\begin{array}{cc}
G_{x} & 0 \\
0 & G_{y}
\end{array}\right) \\
G_{x} & =\left(\begin{array}{cc}
\beta_{x}^{-1 / 2} & 0 \\
\alpha_{x} \beta_{x}^{-1 / 2} & \beta_{x}^{1 / 2}
\end{array}\right) \\
G_{x}^{-1} & =\left(\begin{array}{cc}
\beta_{x}^{1 / 2} & 0 \\
-\alpha_{x} \beta_{x}^{-1 / 2} & \beta_{x}^{-1 / 2}
\end{array}\right) .
\end{aligned}
$$

Since the two matrices are related by the similarity transformation, the eigenvalues and the trace of the matrix are invariant. We futher define a "fundamental" coupling matrix

$$
H=m+n^{+},
$$


where the adjoint operation is the symplectic conjugate operation defined as

$$
n^{+}=-S \tilde{n} S
$$

from which it follows that $n^{+}=n^{-1}$ if $n$ is unimodular. Here $S$ is the unit symplectic matrix.

The roll angle of the eigenmodes is given by

$$
\tan (2 \psi)=\frac{-2 \sqrt{\operatorname{det}(H)}}{\operatorname{Tr}(M-N)}
$$

An important relation for the eigenfrequency is given by

$$
\left(\cos 2 \pi \nu_{1}-\cos 2 \pi \nu_{2}\right)^{2}=\frac{1}{4} \operatorname{Tr}(M-\dot{N})^{2}+\operatorname{det}(H) .
$$

Note that the results in Eqs. (13) and (14) are exact without any approximation. Assuming weak coupling, Peggs shows that the matrices $M$ and $N$ are not perturbed in the first order of the coupler strength. Hence, to the first order approximation,

$$
\operatorname{Tr} M=2 \cos 2 \pi \nu_{x}, \text { and } \operatorname{Tr} N=2 \cos 2 \pi \nu_{y}
$$

Thus, on the coupling resonance $\nu_{x}=\nu_{y}$, Eq. (14) becomes

$$
\delta \nu=\left|\nu_{1}-\nu_{2}\right| \cong \frac{\sqrt{\operatorname{det}(H)}}{2 \pi \sin \pi\left(\nu_{x}+\nu_{y}\right)} .
$$

The procedure to minimize $\delta \nu$ is often called "global decoupling". However, this terminology is misleading. "Global" here does not mean "at all locations of the lattice." The coupling effects on the beam motion which are characterized by the off-diagonal submatrices, $m$ and $n$, of the transfer matrix, $T$, are local and the complete decoupling of the lattice is only possible by removing all the magnets which cause coupling effects.

According to $M$. Billing, $H$ can be written

$$
H=H_{+} \sin \pi\left(\nu_{x}+\nu_{y}\right)+H_{-} \sin \pi\left(\nu_{x}-\nu_{y}\right) \text {. }
$$

$H_{ \pm}$are defined as

$$
H_{ \pm}=\sum_{S Q} q_{m}\left(\begin{array}{rr}
\cos \omega_{ \pm}\left(s_{m}\right) & \sin \omega_{ \pm}\left(s_{m}\right) \\
-\sin \omega_{ \pm}\left(s_{m}\right) & \cos \omega_{ \pm}\left(s_{m}\right)
\end{array}\right)
$$

where $q_{m}=\frac{\sqrt{\beta_{x} \beta_{y}}}{f}$ is the dimensionless skew quadrupole strength of focal length $f$ and

$$
\omega_{ \pm}\left(s_{m}\right)=\left( \pm \phi_{y}\left(s_{m}\right)-\phi_{x}\left(s_{m}\right)\right)+\pi\left( \pm \nu_{y}-\nu_{x}\right)
$$

where $\phi_{x, y}\left(s_{m}\right)$ is the betatron phase at the skew quadrupole measured from the reference point. These expressions are convenient because all the quantities used in the formula are those of the uncoupled lattice. Defining $p=\sum q_{m} \cos \omega_{+}$and $r=\sum q_{m} \sin \omega_{+}, H_{+}$can be 
compactly expressed as

$$
H_{+}=\left(\begin{array}{rr}
p & r \\
-r & p
\end{array}\right) \text {. }
$$

Near the coupling resonance, the contribution of the $H_{-}$term becomes negligibly small. Thus,

$$
\delta \nu=\frac{\sqrt{p^{2}+r^{2}}}{2 \pi}
$$

\subsection{Decoupling Procedure and Its Application}

A routine procedure to decouple the lattice by the operator may be described as follows:

1. Initially, the ring operates at the normal tunes which are $\nu_{x}=35.22$ and $\nu_{y}=14.30$ for the APS storage ring.

2. Adjust the trim quadrupoles to bring the two tunes together as close as possible.

3. Adjust the two families of skew quadrupoles (A:QS and B:QS in the APS storage ring) to minimize the tune separation, $\delta \nu$.

4. Finally, return the tunes to the normal values by adjusting the trim quadrupoles.

The same procedure is simulated using the program MAD interactively. In the simulation, Q1s (see Figure 2 for the location) are chosen as the trim quadrupoles.

Rewriting $p$ and $r$,

$$
\begin{aligned}
p & =\sum_{A: Q S} q_{m} \cos \omega_{+} \sum_{B: Q S} q_{m} \cos \omega_{+}+p_{o} \\
r & =\sum_{A: Q S} q_{m} \sin \omega_{+} \sum_{B: Q S} q_{m} \sin \omega_{+}+r_{o}
\end{aligned}
$$

where $p_{o}$ and $r_{o}$ are from the random roll errors of normal quadrupoles in the ring, we can see that it is convenient to use A:QS to control $r$, and $\mathrm{B}: \mathrm{QS}$ to control $p$, or vice versa. With the midpoint of the straight section of cell 0 as the reference point, we found that A:QS mainly control $r$. With the ten skew quadrupoles of the A:QS family, we get

$$
p(A: Q S)=0.2\left|q_{m}\right|, r(A: Q S)=6.0\left|q_{m}\right| \text {. }
$$

For optimal control of $p$ using the B:QS family, we consult Fig. 3 (top) in order to find the most efficient arrangement. There we find that the phase of $n=4$ or $n=9$ arrangement is almost orthogonal to that of A:QS. The relevance of using Fig. 3 (top) may be traced to the definition of $\omega_{+}$,

$$
\omega_{+}=\phi_{y}\left(s_{m}\right)-\phi_{x}\left(s_{m}\right)=\left(\nu_{y}-\nu_{x}\right) \theta_{m}=21 \theta_{m}
$$

In the simulation, we used the $n=0$ arrangement of B:QS. The coupling ratio due to the random roll errors of normal quadrupoles is shown in Fig. 5 for three different error 


\begin{tabular}{|c|c|c|c|c|}
\hline Error level & $\delta \nu$ (before) & $\delta \nu$ (after) & $\mathrm{A}: \mathrm{QS}\left(\mathrm{B}^{\prime} \mathrm{l}\right)$ & $\mathrm{B}: \mathrm{QS}$ (B') \\
\hline $0.5 \mathrm{mrad}$ & 0.0186 & 0.00133 & $0.019 \mathrm{~T}$ & $0.055 \mathrm{~T}$ \\
\hline $1.0 \mathrm{mrad}$ & 0.0353 & 0.00465 & $0.031 \mathrm{~T}$ & $0.100 \mathrm{~T}$ \\
\hline $2.0 \mathrm{mrad}$ & 0.0714 & 0.0282 & $0.019 \mathrm{~T}$ & $0.140 \mathrm{~T}$ \\
\hline
\end{tabular}

Table 3: Effect of Decoupling Procedure on the Tunes

levels ( $0.5 \mathrm{mrad}, 1 \mathrm{mrad}$, and $2 \mathrm{mrad})$. The minimum tune separations before and after decoupling are summarized in Table 3 with the same seed number for the assignment of random errors. We note that $\delta \nu$ before decoupling is linearly proportional to the magnitude of rms errors as expected.

Finally, the effects of decoupling on the phase motion at the normal tunes, shown in Fig. 6 for the error level of $2 \mathrm{mrad}$, indicate that our decoupling procedure effectively reduced the vertical emittance. The turn by turn single particle emittances are also plotted (see Fig. 7).

\section{Conclusion}

In this report we investigated the introduction of coupling to put a beam in the fully coupled state and the decoupling procedure to cancel the coupling effects due to the random roll errors of normal quadrupoles. The harmonic analysis of skew quadrupole distribution provides the common ground for finding the optimum arrangement of skew quadrupoles. We achieved full coupling at the integrated skew quadrupole strength of $0.2 \mathrm{~T}$ and we reduced the coupling by the decoupling procedure down to 10 percent even with the rather large rms roll error of 2 mrad.

\section{Acknowledgement}

This work was initiated by Glenn Decker and discussions with E. Crosbie and L. Emery were helpful.

\section{Reference}

1. K. Symon, "Applied Hamiltonian Dynamics," unpublished lecture note for 1992 U.S. Particle Accelerator Summer School, October, 1991.

2. H. Bruck, "Circular Particle Accelerator," LA-TR-72-10.

3. G. Decker, "Nomenclature and Name Assignment Rules for the APS Storage Ring," ANL/APS/LS-196, March, 1992.

4. K.L. Brown and R.V. Servranckx, "Cross-Plane Coupling and Its Effects on Projected Emittance," SLAC-PUB-4679, August, 1989. 
5. H. Grote and F.C. Iselin, "The MAD Program Version 8.1," CERN/SL/90-13(AP), May, 1990.

6. F. Iazzourene, et al., "RACETRACK User's Guide Version 4," Sincrotrone Trieste, July, 1991.

7. D. Edwards and L.C. Teng, "Parameterization of Linear Coupled Motion in Periodic Systems," IEEE NS-30, No. 3, June, 1973.

8. S. Peggs, "Coupling and Decoupling in Storage Rings," IEEE NS-30, No. 4, August, 1983.

9. R. Talman, "Single Particle Motion," Frontiers of Particle Beams, Lecture Notes in Physics, Vol. 343, Springer-Verlag.

10. M. Billing, "Controls in Use at CESR for Adjusting Horizontal to Vertical Coupling," IEEE NS-32, No. 5, October, 1985.

11. P. Bagley and D. Rubin, "Diagnosis of Transverse Coupling Errors in a Storage Ring," Proc. of the 1987 Particle Accelerator Conf., pp. 1301.

12. P. Bagley and D. Rubin, "Correction of Transverse Coupling in a Storage Ring," Proc. of the 1989 Particle Accelerator Conf., pp. 874.

13. L. Schachinger, "Interactive Global Decoupling of the SSC Injection Lattice ," SSC Central Design Group Report SSC-N-433, December, 1987. 

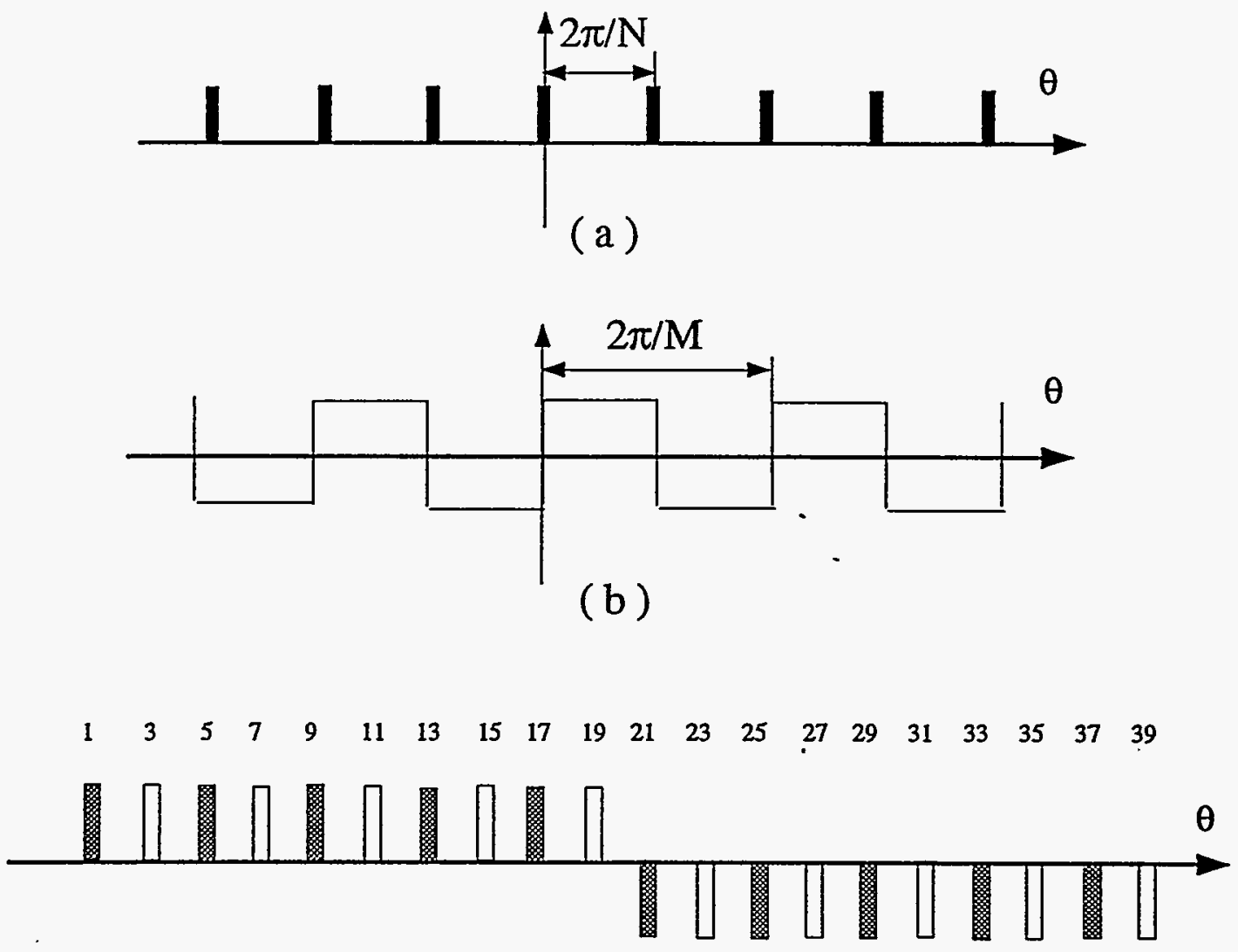

(c)

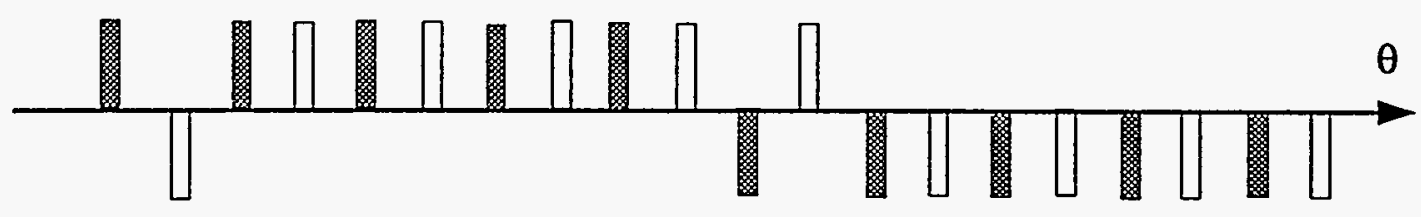

(d)

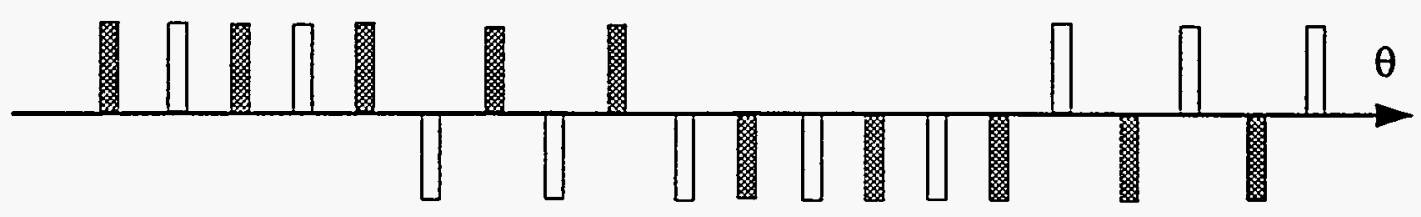

(e)

Fig. 1. (a) Periodic delta function, (b) Staircase function, (c) Skew quadrupole arrangement solid block-A:QS, blank block-B:QS, (d) Shift of A:QS, $n=1$, (e) Shift of B:QS, $n=7$ (the optimized arrangement achiving full coupling). 


\section{Storage Ring Sector and \\ Magnet Nomenclature}

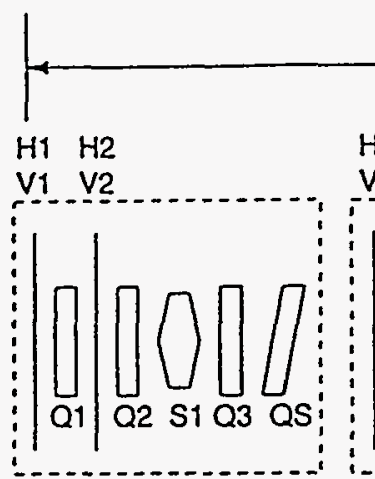

A

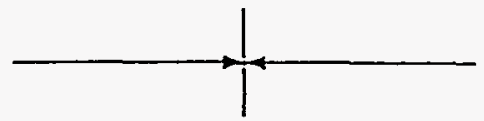

B

V3
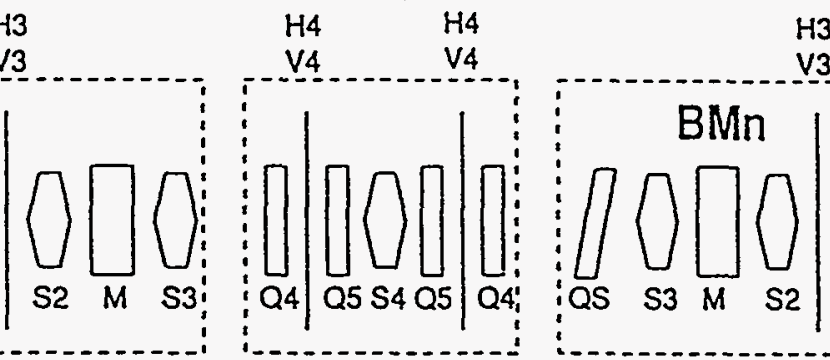

$\mathrm{H} 2 \mathrm{H} 1$

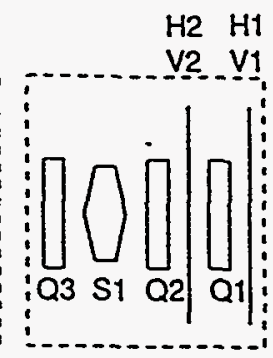

IDn

Girder 1

Girder 2

Girder 3

Girder 4

Girder 5

$S(n) A:(H, V) 1$

S(n)A:Q1

$S(n) A: P 1$

$S(n) A:(H, V) 2$

S(n)A:Q2

S(n)A:P2

$S(n) A . S 1$

S(n)A:Q3

$S(n) A: Q S$

S(n)A:Q4
S(n)A:(H,V)4
S(n)A:Q5
S(n)A:S4
S(n)B:P5
S(n)B:Q5
S(n)B:(H,V)4
S(n)B:Q4

S(n)B:QS

$S(n) B: S 3$

$S(n) B: P 4$

$S(n) B: M$

$S(n) B: P 3$

$S(n) B: S 2$

$S(n) B:(H, V) 3$

$S(n) B: Q 3$

$S(n) B: S 1$

$S(n) B: P 2$

$S(n) B: Q 2$

S(n)A:P4

$S(n) A: S 3$

$\mathrm{S}(\mathrm{n}) \mathrm{B}: \mathrm{Q} 4$

$S(n) B:(H, V) 2$

$S(n) B: P 1$

S(n)B:Q1

$S(n) B:(H, V) 1$

$\mathrm{S}=$ Storage Ring

$n=$ Sector Number

$A, B=$ Upstream, Downstream Half of Sector

$:=$ Delimiter - Do not use period (.)

Q1-Q5 = Quadrupoles

QS = Skew Quadrupole

S1-S4 = Sextupoles

$\mathrm{H} 1-\mathrm{H} 4=$ Horizontal Steering Correctors

V1-V4 = Vertical Steering Correctors

$\mathrm{M}=$ Dipoles

$P=$ Position Monitors

ID = Insertion Device Source

$\mathrm{BM}=$ Bending Magnet Source

Fig. 2. Nomenclature for the APS storage ring. (From G. Decker, Ref. [3]) 

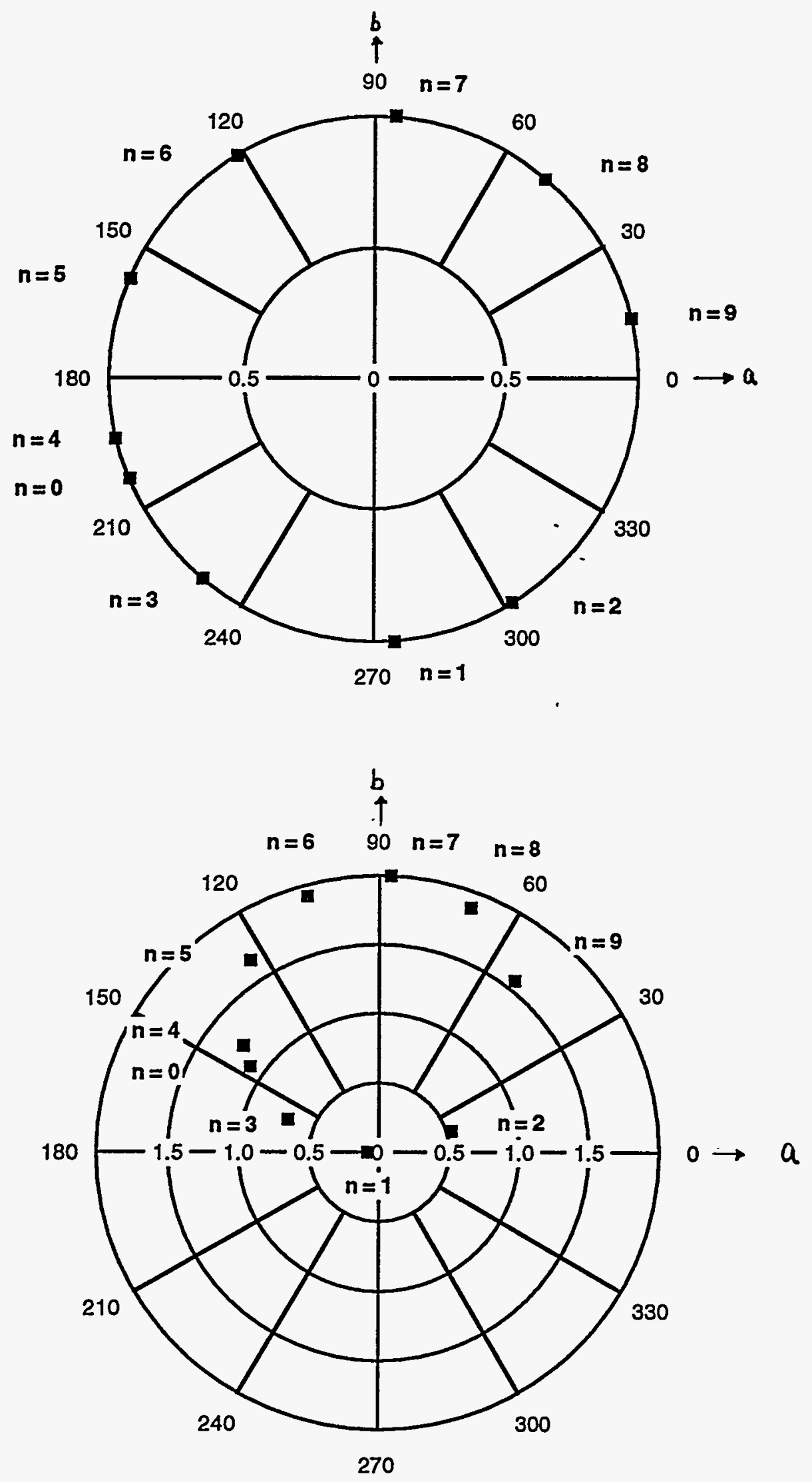

Fig. 3. (Top) $a$ and $b$ coefficients from the B:QS skew quadrupole family,

(Bottom) total $a$ and $b$ coefficients including both the A:QS and the B:QS skew quadrupole families. 

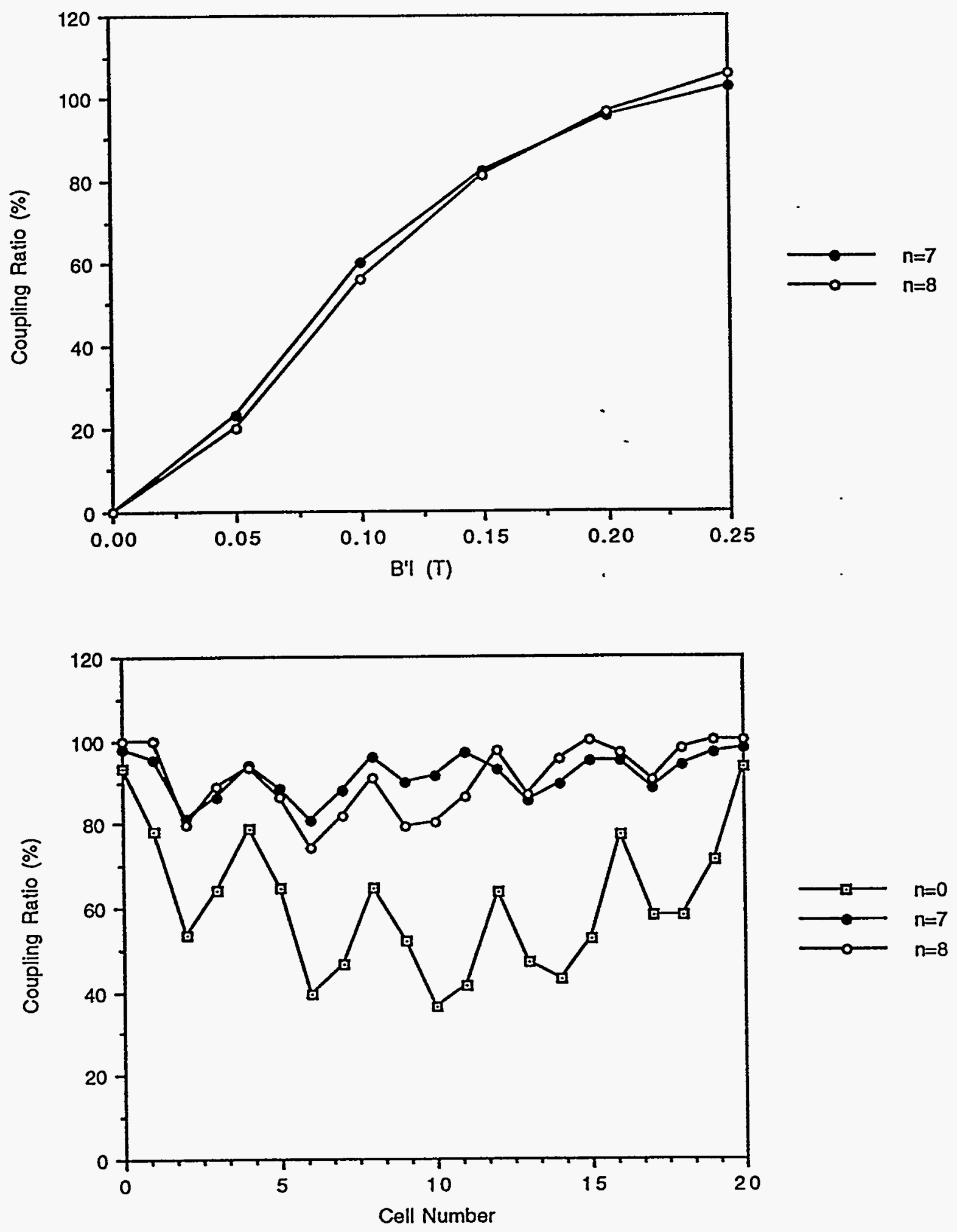

Fig. 4. (Top) Coupling Ratio vs B'l (T), (Bottom) Coupling Ratio Around Ring. 


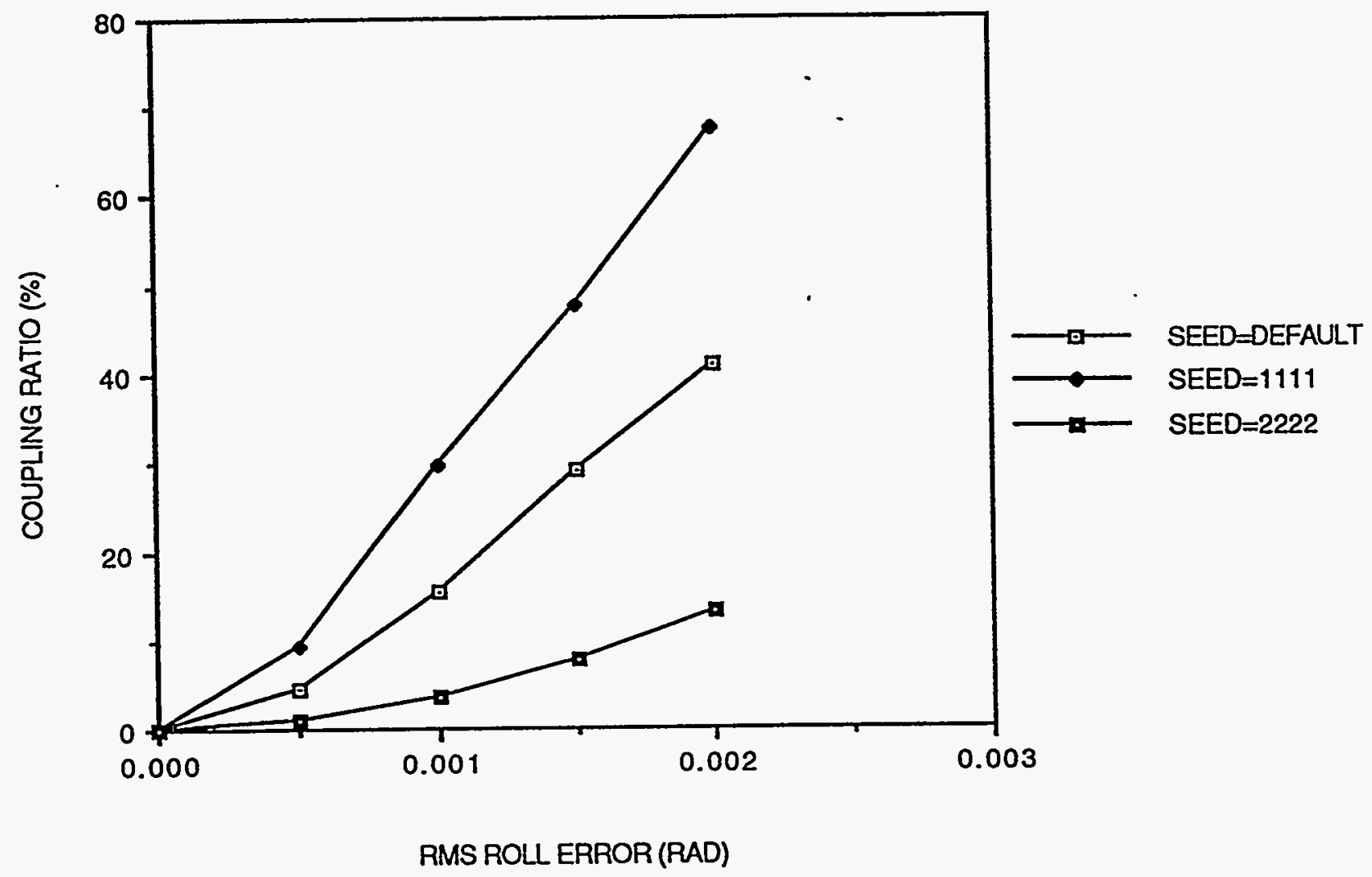

Fig. 5. Coupling ratio due to the random roll errors of normal quadrupoles. 

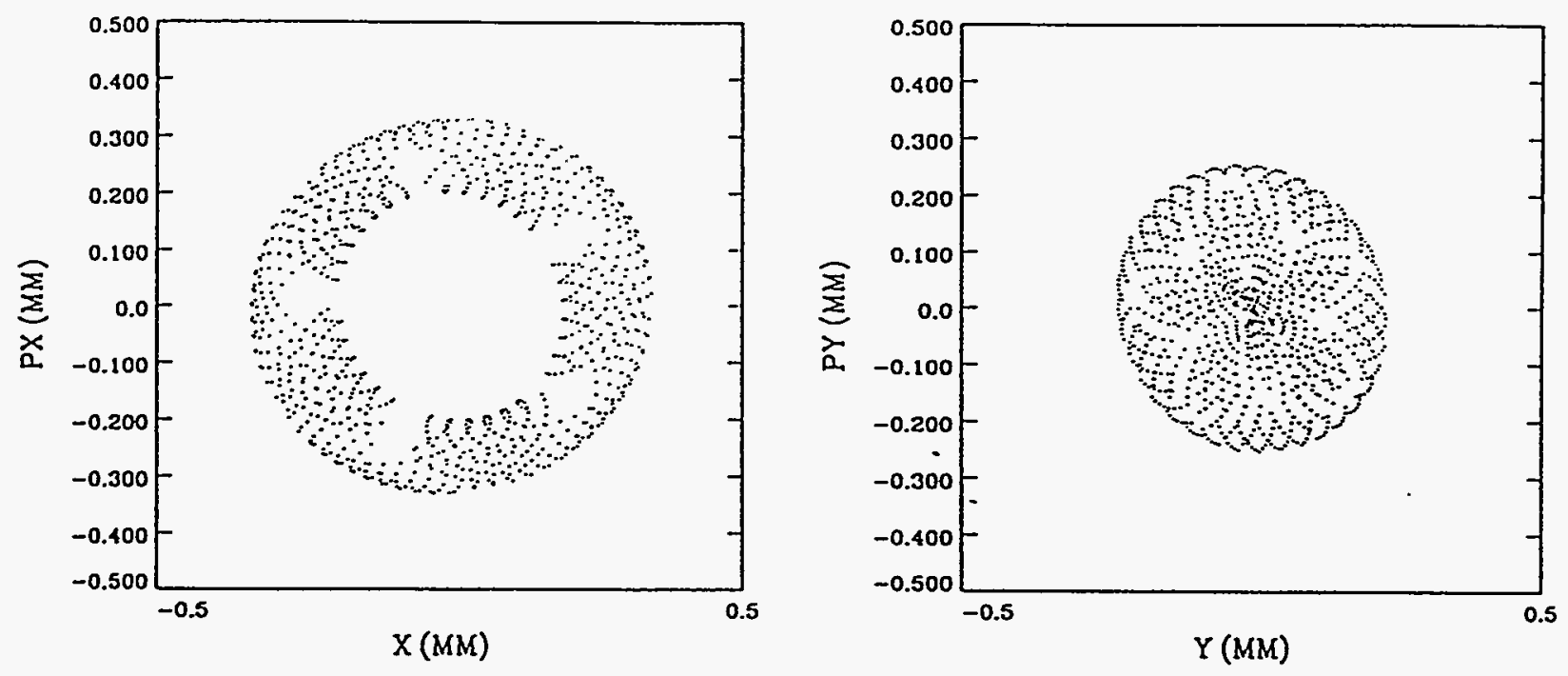

HORIZONTAL PHASE SPACE

VERTICAL PHASE SPACE
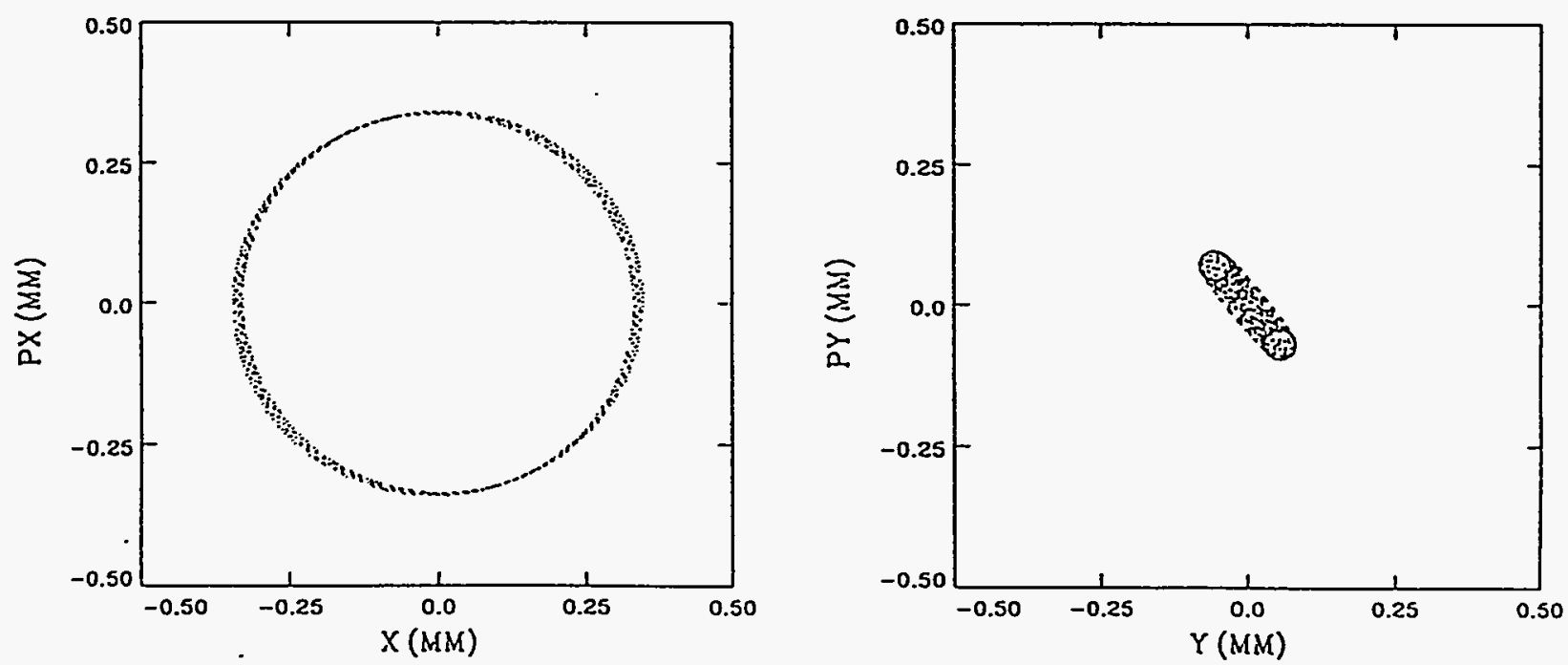

Fig. 6. The effects of decoupling on the phase motion. (Top) Before decoupling, (Bottom) After decoupling. 
HORIZONTAL EMITTANCE

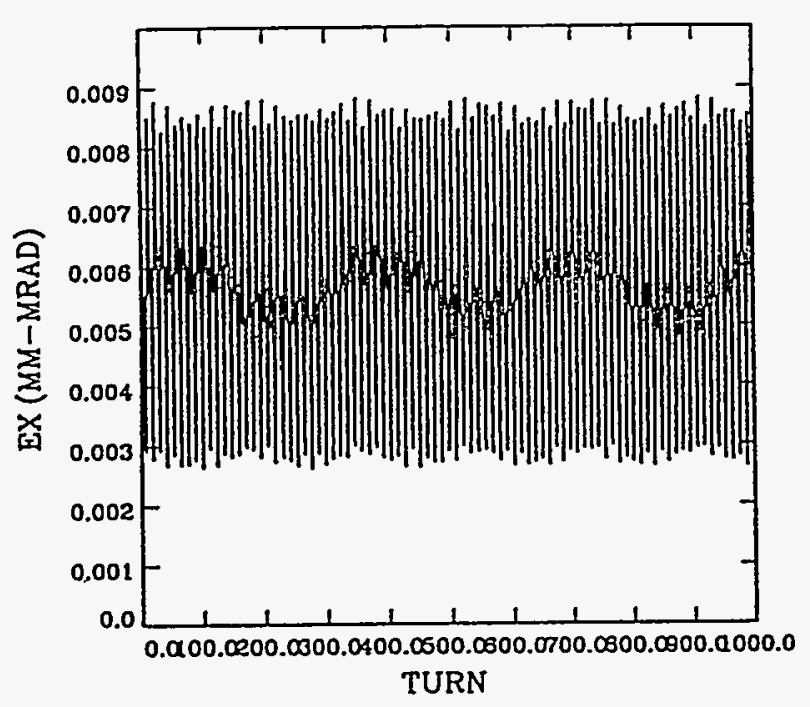

HORIZONTAL EMITTANCE

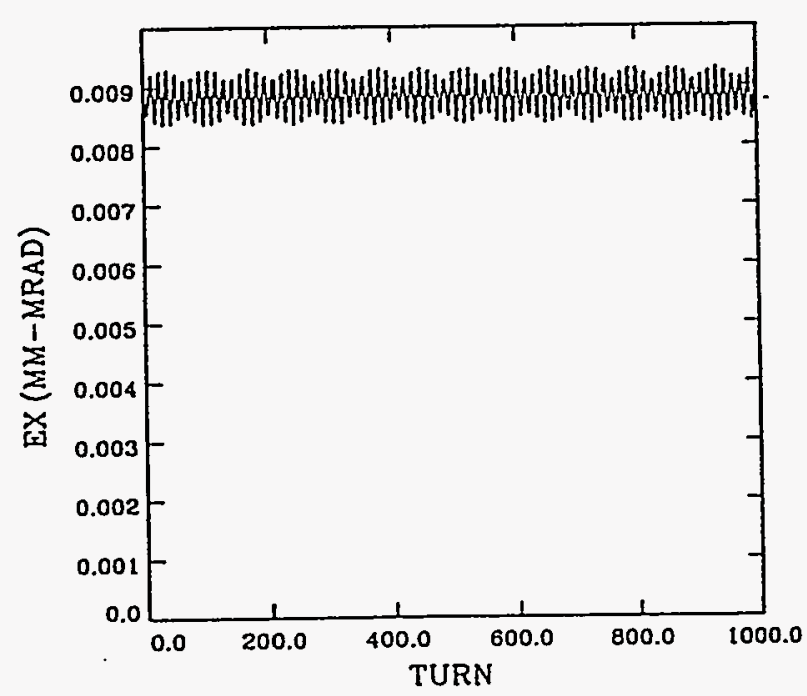

VERTICAL EMITTANCE

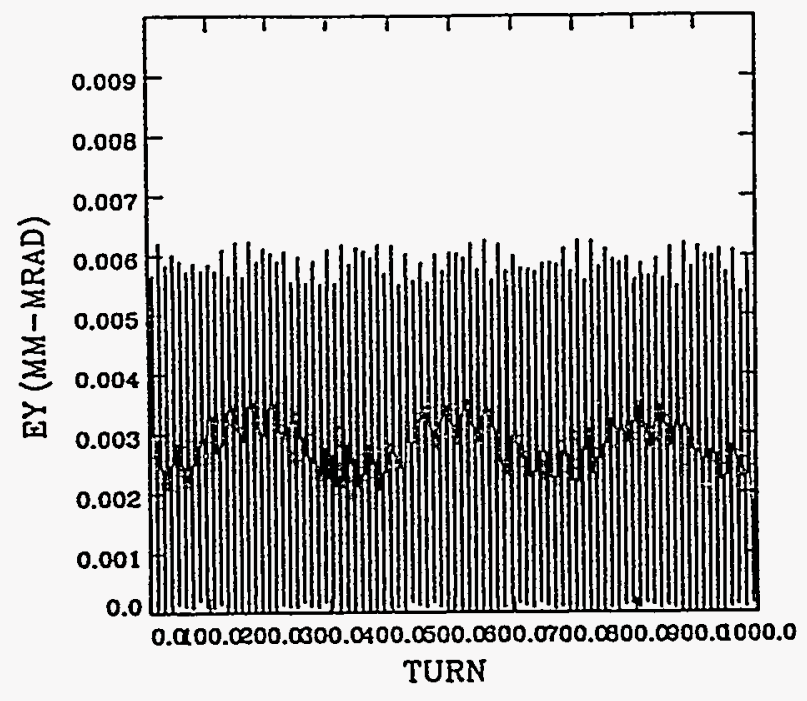

VERTICAL EMITTANCE

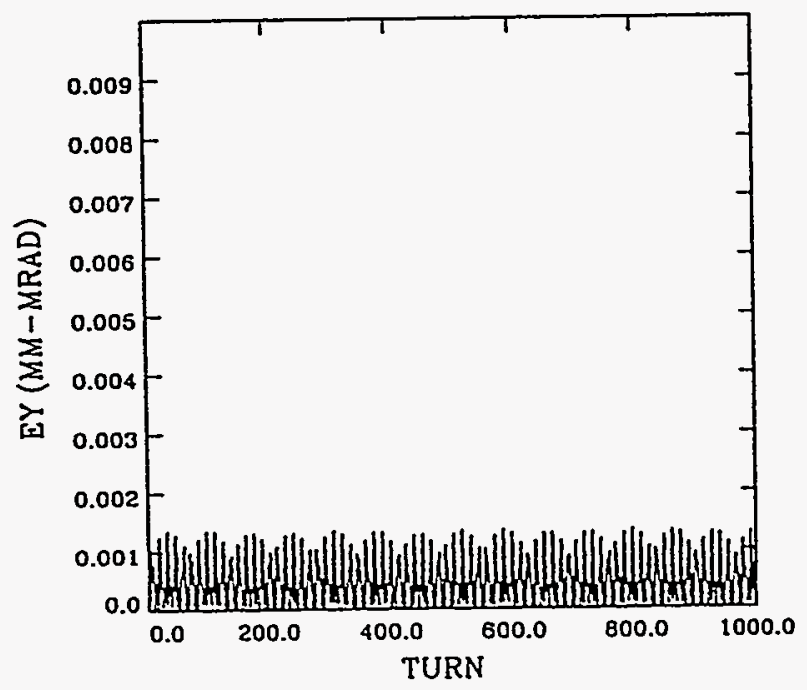

Fig. 7. The effects of decoupling on the emittance.

(Top) Before decoupling, (Bottom) After decoupling. 